\title{
Troubleshooting Complications of Intrathecal Baclofen Therapy
}

Stevenson $\mathrm{VL}^{1,2 *}$, Keenan $\mathrm{E}^{1}$ and $\mathrm{J}$ arrett $\mathrm{L}^{3}$

${ }^{1}$ National Hospital for Neurology and Neurosurgery, UCLH NHS Foundation Trust, London, UK

${ }^{2}$ University College London, Institute of Neurology, London, UK

${ }^{3}$ Royal Devon and Exeter NHS Foundation Trust, Exeter, UK

*Corresponding author: Valerie Stevenson, Box 113, The National Hospital for Neurology and Neurosurgery, Queen Square, London, WC1N 3BG, UK

Received: September 25, 2017; Accepted: October 17, 2017; Published: October 24, 2017

\begin{abstract}
Intrathecal baclofen (ITB) is a well-recognised treatment for severe spasticity refractory to oral medications. However it is known to have a significant complication rate with serious potentially fatal consequences of baclofen overdose or withdrawal. It is therefore essential that patients receiving ITB therapy are managed by responsive, accessible multidisciplinary services with a systematic and timely approach to troubleshooting complications when they occur. A troubleshooting algorithm is presented here to facilitate prompt and effective investigations and treatment; this tool can be adapted by individual services to suit their own particular patient cohort and healthcare setting.
\end{abstract}

Keywords: Intrathecal baclofen; Troubleshooting; Algorithm; Complications; Spasticity

\section{Introduction}

Intrathecal baclofen (ITB) was first used in 1985 for spinal cord injury [1] and has since been shown to be an effective treatment in the management of severe spasticity of either cerebral or spinal origin [2-5] and in monophasic or progressive conditions [6,7].

In long-term follow-up studies, the benefit has proved to be sustainable over time [8-11], with many individuals demonstrating high levels of satisfaction and continuing to benefit following a pump replacement once the battery life of the original pump has been depleted.

Use of intrathecal baclofen requires a coordinated approach by an experienced multidisciplinary team including a neurologist or rehabilitation physician, neurosurgeon, physiotherapists, nurses, and occupational therapists. The process involves careful patient selection, detailed assessment including a trial of ITB, implantation and importantly both responsive and accessible follow up as ITB therapy is not without risk of complications. Whenever complications are suspected investigation and treatment should be instigated promptly and in a systematic way [1].

Complication rates of ITB therapy vary between published studies depending on their definition of adverse events, the population studied and the follow up period, rates are however not insignificant and range between 4 and $25 \%$. Complications can be considered as mechanical (ie a hardware issue with the pump or catheter), infection related, drug or procedure related (for example baclofen overdose or deep vein thrombosis). Mechanical complications are the most frequently observed; these usually involve catheter malfunction (disconnection, kinks, breaks or displacement) [13-15]. Pump dysfunction is rare with the use of baclofen but corrosion of the internal tubing causing a motor stall can occur particularly if pumps are used off label with drug mixtures [16]. Data reported from Medtronic through their Implantable Systems Performance Registry on 7,459 patients with pumps $(21.6 \%$ for intractable spasticity) revealed a total of 1,393 product performance events in 982 patients enrolled (13.17\%), $75.7 \%$ of these were related to catheter malfunction [17].

\section{Investigation of potential system malfunction}

If there is any suspicion of ITB system dysfunction (symptoms of over or under dosage or failure to gain control of spasticity on escalating dosages) it is important to investigate promptly to avoid potentially fatal withdrawal syndromes. There is no agreed consensus on the process used for investigation; previously published algorithms have looked at different aspects including troubleshooting in the outpatient setting, managing ITB withdrawal and overdose as well as extensive investigations of the pump system [18-20].

We present our troubleshooting algorithm illustrated in Figure 1 which is based on the author's own experience of ITB practice and takes a pragmatic approach ensuring investigation is sufficient to ensure confidence in the need for surgical intervention but also prevents delay in diagnosing mechanical complications. The National Hospital for Neurology and Neurosurgery, University College London Hospitals has been utilising ITB since 1994 (currently 155 patients under ITB pump follow up). The choice of particular investigations and the order they are used will of course be influenced by investigation availability and the particular nuances of the patient concerned. Other investigations not included in our algorithm, such as magnetic resonance imaging (MRI) or radio-isotope scintigraphy [21], are available however on a pragmatic note the demonstration of a non-functioning catheter requires surgical replacement and there is therefore often no need to proceed to extensive investigation and imaging.

The first stage of investigation when suspecting ITB system malfunction is to take a careful history exploring the presence of, and addressing, any noxious stimulus (for example infection, concomitant medication effects, pressure sores, bladder and bowel dysfunction, undiagnosed fractures or deep vein thrombosis and importantly progression/remission of underlying neurological disorder). It is also important to explore whether there are any precipitants to ITB treatment failure such as recent falls, MRI, or rarer causes of pump malfunction such as high dose radiation, SCUBA diving, use of 


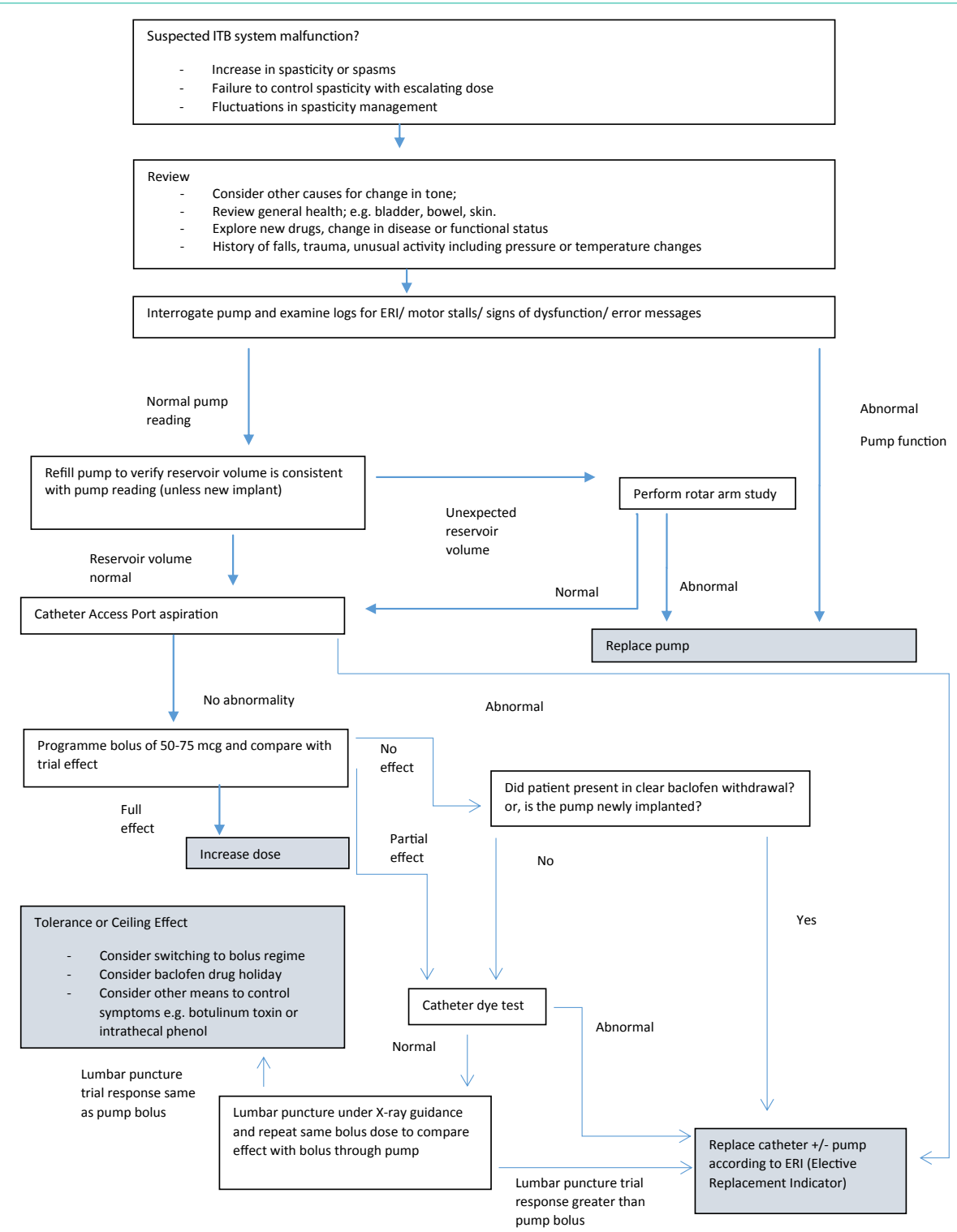

Figure 1: Algorithm for ITB troubleshooting. Adapted from Stevenson VL. Intrathecal baclofen and phenol: Spasticity Management, A Practical Multidisciplinary Guide. $2^{\text {nd }}$ ed. Taylor and Francis. 2016; 129-160 [12].

hyperbaric oxygen chambers or vibration plates [12].

If these factors are excluded or managed and spasticity remains problematic then it is vital to proceed swiftly to pump system investigation.

\section{Pump interrogation and reservoir volume verification}

Electronic pumps have alarms to alert if any problems are occurring although these can sometimes be overlooked by patients or their carers. Whether or not an alarm is sounding, the electronic pump status can be checked by reading the pump with the programmer; this will identify the nature of any pump problem. In addition, an ongoing regular check for pump efficiency can be made when the reservoir is refilled. The actual volume of baclofen removed from the reservoir can be checked against the expected amount: more or less than the expected volume (outside of accepted tolerances of $\sim 15 \%$ ) could indicate an over- or under-infusing pump.

\section{Bolus dose testing}

The pump can be programmed to deliver a bolus equivalent to a trial test dose (usually $50-75 \mathrm{mcg}$ ) over a few minutes. Hence the original ITB trial can be reproduced and outcome measures assessed at 4 hours. If the system is functioning the effect should mimic the trial, particularly if this is used in the early post-implantation period to assess for surgical problems at implantation. If loss of baclofen effect occurs several years after implantation or the effect of a programmed bolus is partial it is sometimes useful to perform a bolus via lumbar puncture (taking care not to puncture the catheter; $\mathrm{X}$-ray or ultrasound can be useful for this) at the same dose as the pump programmed bolus to compare the response. A difference in effect implies a catheter leak necessitating surgical replacement of the catheter, where as an equal effect suggests either a ceiling effect (no additional benefit on escalating doses) or rarely the development of baclofen tolerance (the effect ameliorating over time). 


\section{X-ray}

It is important to note that many intrathecal catheters currently in use are not radio-opaque and only have a catheter tip marker; plain $\mathrm{X}$-rays therefore have a limited role to play in investigating suspected ITB failure. With radio-opaque catheters a disconnection or fracture may however be visible excluding the need for further investigations.

\section{Rotar arm (pump roller) study}

With the Medtronic Synchromed II pump it is possible to perform a plain film of the pump then programme a specific bolus of ITB and repeat the X-ray, this demonstrates the rotar arm is moving appropriately and excludes pump dysfunction as a cause for baclofen inefficacy. However, if there is no discrepancy with reservoir volumes and the pump telemetry shows a normally functioning pump this is usually unnecessary to perform.

\section{Catheter aspiration and radio-opaque dye study}

If the pump has a catheter access port the catheter can be aspirated to check for CSF flow; 3-4mls should be aspirated. Difficulty aspirating or bubbles indicate a catheter problem and surgical exploration with catheter reconnection or replacement is indicated.

If the flow is unimpeded and CSF can be readily withdrawn a radiopaque dye study can be performed, this may identify whether baclofen is leaking from the catheter or if it has become displaced into the epidural space. It is important to ensure that the catheter has been fully emptied before injecting contrast: otherwise a bolus of baclofen will be delivered with the dye potentially leading to an overdose. Unfortunately there is a high false negative rate with dye studies because the high pressure and volume of contrast injected can easily bypass fractures/ micro-tears and overcome kinks.

\section{Computed tomography (CT)}

CT may provide more detail and can be combined with the catheter dye study; this is particularly helpful in delineating whether contrast is in the intrathecal or epidural space, however it is not always readily available [22].

\section{Conclusion}

There is no doubt that intrathecal baclofen is an effective treatment to manage severe lower limb spasticity and spasms, however complications do occur and if not managed appropriately can result in significant morbidity or mortality. It is therefore essential that all patients receiving ITB therapy are managed in a responsive, accessible multidisciplinary service with a systematic and timely approach to troubleshooting complications when they occur. The algorithm presented here is a tool for services to use or adapt to suit their own particular patient cohort and healthcare setting.

\section{References}

1. Penn RD, Kroin JS. Continuous intrathecal baclofen for severe spasticity Lancet. 1985; 2: 125-127.

2. Albright A, Cervi A, Singletary J. Intrathecal baclofen for spasticity in cerebral palsy. JAMA. 1991; 265: 1418-1422.

3. Meythaler JM, McCary A, Hadley M. Prospective assessment of continuous intrathecal infusion of baclofen for spasticity caused by acquired brain injury: A preliminary report. J Neurosurg. 1997; 87: 415-419.
4. Loubser PG, Narayan RK, Sandin KJ, et al. Continuous infusion of intrathecal baclofen: Longterm effects on spasticity in spinal cord injury. Paraplegia. 1991; 29: 48-64.

5. Natale M, Mirone G, Rotondo M, Moraci A. Intrathecal baclofen therapy for severe spasticity: Analysis on a series of 112 consecutive patients and future prospectives. Clin Neurol Neurosurg. 2012; 114: 321-325.

6. Dan B, Motta F, Vles JS, et al. Consensus on the appropriate use of intrathecal baclofen (ITB) therapy in paediatric spasticity. Eur J Paediatr Neurol. 2010; 14: 19-28.

7. Bonouvrie LA, van Schie PEM, Becher JG, van Ouwerkerk WJR, Vermeulen RJ. Intrathecal baclofen for progressive neurological disease in childhood: A systematic review of the literature. Eur J Paediatr Neurol. 2012; 16: 279-284.

8. Vles GF, Soudant DL, Hoving MA, et al. Long-term follow-up on continuous intrathecal baclofen therapy in nonambulant children with intractable spastic cerebral palsy. Eur J Paediatr Neurol. 2013; 17: 639-644.

9. Draulans N, Vermeersch K, Degraeuwe B, et al. Intrathecal baclofen in multiple sclerosis and spinal cord injury: Complications and long-term dosage evolution. Clin Rehabil. 2013; 27: 1137-1143.

10. Zahavi A, Geertzen JHB, Middel B, et al. Long term effect (more than five years) of intrathecal baclofen on impairment, disability and quality of life in patients with severe spasticity of spinal origin. J Neurol Neurosurg Psychiatry. 2004; 75: 1553-1557.

11. Mathur SN, Chu SK, McCormick Z, Chang Chien GC, Marciniak CM. Longterm intrathecal baclofen: Outcomes after more than 10 years of treatment. PMR. 2014; 6: 506-513.

12. Stevenson VL. Intrathecal baclofen and phenol: Spasticity Management, A Practical Multidisciplinary Guide. $2^{\text {nd }}$ ed. Taylor and Francis. 2016; 129-160.

13. Motta F, Antonello CE. Analysis of complications in 430 consecutive pediatric patients treated with intrathecal baclofen therapy: 14-year experience. J Neurosurg Pediatr. 2014; 13: 301-306.

14. Borrini L, Bensmail D, Thiebaut JB, Hugeron C, Rech C, Jourdan C. Occurrence of adverse events in long-term intrathecal baclofen infusion: A 1-year follow-up study of 158 adults. Arch Phys Med Rehabil. 2014; 95: 10321038.

15. Ghosh D, Mainali G, Khera J, Luciano M. Complications of intrathecal baclofen pumps in children: Experience from a tertiary care center. Pediatr Neurosurg. 2013; 49: 138-144.

16. Riordan J, Murphy P. Intrathecal pump: An abrupt intermittent pump failure. Neuromodulation. 2015; 18: 433-435.

17. Medtronic Product Performance Report. 2016.

18. Boster A, Nicholas J, Bartoszek MP, O'Connell C, Oluigbo C. Managing loss of intrathecal baclofen efficacy: Review of the literature and proposed troubleshooting algorithm. Neurol Clin Pract. 2014; 4: 123-130.

19. Dvorak EM, McGuire JR, Nelson ME. Incidence and identification of intrathecal baclofen catheter malfunction. PMR. 2010; 2: 751-756.

20. Saulino M, Anderson DJ, Doble J, Farid R, Gul F, Konrad P, Boster AL. Best Practices for IntrathecalBaclofen Therapy: Troubleshooting. Neuromodulation. 2016; 19: 632-641.

21. Miracle AC, Fox MA, Ayyangar RN, Vyas A, Mukherji SK, Quint DJ. Imaging evaluation of intrathecal baclofen pump-catheter systems. AJNR Am J Neuroradiol. 2011; 32: 1158-1164.

22. Schapiro A, Racadio J, Kinnett D, Maugans T. Combined C-arm fluoroscopy and C-arm cone beam computed tomography for the evaluation of patients with possible intrathecal baclofen delivery system malfunctions. Neurosurgery. 2011; 69: 27-33; discussion on s33. 